\title{
Social and Psychological Factors in Bilingual Speech Production: Introduction to the Special Issue
}

\author{
Robert Mayr $^{1, *(1)}$ and Jonathan Morris ${ }^{2}$ \\ 1 Centre for Speech and Language Therapy and Hearing Science, Cardiff Metropolitan University, \\ Llandaff Campus, Western Avenue, Cardiff CF5 2YB, UK \\ 2 School of Welsh, Cardiff University, Colum Drive, Cardiff CF10 3EU, UK; morrisj17@cardiff.ac.uk \\ * Correspondence: rmayr@cardiffmet.ac.uk
}

check for

updates

Citation: Mayr, Robert, and Jonathan Morris. 2021. Social and

Psychological Factors in Bilingual Speech Production: Introduction to the Special Issue. Languages 6: 155. https: / / doi.org/10.3390/

languages6040155

Received: 14 September 2021

Accepted: 24 September 2021

Published: 28 September 2021

Publisher's Note: MDPI stays neutral with regard to jurisdictional claims in published maps and institutional affiliations.

Copyright: (c) 2021 by the authors. Licensee MDPI, Basel, Switzerland. This article is an open access article distributed under the terms and conditions of the Creative Commons Attribution (CC BY) license (https:// creativecommons.org/licenses/by/ $4.0 /)$.
The pronunciation patterns of bilinguals and their development have been investigated in a number of ways. Generally, a distinction is made between internal linguistic factors, on the one hand, and external social and psychological factors, on the other. Linguistic factors that have been identified as critically affecting L1 and L2 accentual targets include cross-linguistic interactions and language transfer; language universals and markedness; and the similarity of L1 and L2 sounds. The study of linguistic factors contributes significantly to our understanding of the mechanisms that underpin bilingual speech processing and development. This is reflected in the central role that such factors play within current theoretical models in bilingual speech research, such as the revised Speech Learning Model, SLM-r (Flege and Bohn 2021); the Perceptual Assimilation Model of Second Language Learning, PAM-L2 (Best and Tyler 2007); and the Second Language Linguistic Perception Model, L2LP (Escudero 2005).

Importantly, however, these internal factors cannot fully explain the bilingual speech patterns we observe: they are critically mediated by a myriad of extra-linguistic cognitive and psychological factors, such as L1 and L2 use, age of onset of learning, length of residence in an L2-speaking environment or motivation, to name but a few (see, e.g., Piske et al. (2001) for an overview). Moreover, recent variationist and experimental research has documented the significance of social factors, such as peer group identity or cultural and ethnic orientation, on the speech patterns of bilinguals, in particular in the context of long-term contact and minority language bilingualism (Mayr et al. 2017; Nance 2020; Sharma and Sankaran 2011).

The present Special Issue sought to deepen our understanding of such extra-linguistic variables by bringing together ten state-of-the-art articles that investigate the role of one or several social and/or psychological factors on the speech patterns of bilingual speakers. The articles feature a wide range of bilingual populations and contexts-from child and adult bilinguals in heritage language settings to adult new speakers in bilingual societies, and L2 learners and L1 attriters in migration contexts-and encompass a variety of examined languages, including Arabic, English, Galician, German, Italian, Portuguese, Russian, Spanish and Welsh. They are diverse in methodological terms, from auditory and acoustic analyses of bilinguals' speech patterns to accent identification tasks and online questionnaires, and aim to inform current theoretical debates. Contributors are leading experts from universities in Austria, Canada, England, Germany, Italy, Norway, Oman, the United States and Wales.

The ten articles in the Special Issue have been organized thematically. As a result, the order presented here differs somewhat from that of the articles published on the journal website. Specifically, it commences with two articles on child bilinguals in heritage language settings (Montanari, Mayr and Subrahmanyam; Kupisch, Kolb, Rodina and Urek), followed by an article on the speech patterns of adult heritage speakers across generations (Baird, Cristiano and Nagy). The subsequent three papers, in turn, are situated 
within the context of minority languages in bilingual societies (Tomé Lourido and Evans; Williams and Cooper; Morris), with specific focus on the accents of new speakers. Finally, the Special Issue concludes with four articles that focus on adult L2 speech acquisition and/ or the effects of the L2 experience on L1 speech patterns (Al-Kendi and Khattab; Reubold, Diteweg, Mayr and Mennen; Osborne and Simonet; Kornder and Mennen). In what follows, details of each of these are presented in turn.

The study by Simona Montanari (California State University, Los Angeles, Robert Mayr (Cardiff Metropolitan University) and Kaveri Subrahmanyam (California State University, Los Angeles) entitled "Maternal cultural orientation and speech sound production in Spanish/English dual language preschoolers" examines the effect of maternal orientation to Anglo-American (acculturation) and Mexican (enculturation) culture on the speech patterns of Spanish-English bilingual children from low socio-economic backgrounds growing up in Spanish-speaking homes in Southern California. To this end, single-word samples were elicited from the children in Spanish and English, using picture prompts, that together encompass the consonant and vowel categories of the two languages. Mothers' acculturation and enculturation levels, in turn, were assessed on the basis of a rating scale. The results revealed a significant correlation between maternal acculturation and children's segmental accuracy in English, that is, the greater the mothers' orientation towards AngloAmerican culture, the more accurately their children produced English consonants and vowels. No such relation was found for children's accuracy on Spanish segments. The authors interpret these findings as arising from more American-oriented mothers using English more with their children, which may have reinforced their phonological skills in the language. The lack of a correlation for Spanish, on the other hand, is likely due to consistently high levels of Mexican orientation amongst the mothers in the sample or language input differences due to different social practices.

The second article featuring child bilinguals, "Foreign accent in pre- and primary school heritage bilinguals" by Tanja Kupisch (Universität Konstanz and UiT, The Arctic University of Norway), Nadine Kolb (UiT, The Arctic University of Norway), Yulia Rodina (UiT, The Arctic University of Norway) and Olga Urek (UiT, The Arctic University of Norway), investigates the effects of age in the perceived accent of German-Russian bilingual children in Germany alongside native controls in both languages. Using a global accent rating task, samples of speech from the children's narratives in both languages were rated by native Russian and German listeners. The results revealed that the bilinguals were perceived as foreign-accented in both languages more often than the monolingual controls. Moreover, the older bilinguals' accent was perceived as more authentic in German than that of the younger bilinguals, while the reverse was true for Russian. These effects were enhanced in families with two Russian parents compared with those with only one Russian-speaking parent. The authors conclude that the primary school years are critical for the maintenance of the heritage language as it comes under pressure from the majority language.

The third article, "Apocope in heritage Italian" by Anissa Baird (University of Toronto), Angela Cristiano (Università di Bologna) and Naomi Nagy (University of Toronto), examines whether three generations of Calabrian Italian heritage speakers in Toronto delete (apocope) or reduce word-final vowels in their heritage language in line with the patterns seen in southern Italian varieties. Using sociolinguistic interviews, word-final vowel productions from the heritage speakers and a homeland comparator group were analyzed auditorily and subsequently related to a large number of predictor variables, including linguistic and social ones, using mixed effects regression models. The results showed that the heritage speakers made use of both apocope and reduction. While their distributions were similar to those of homeland speakers, some inter-generational differences occurred. Both apocope and reduction were found to be primarily influenced by linguistic factors, while surprisingly few social factors mediated the use of these socially marked forms. Together, the findings of the study reveal a complex interplay of social and linguistic factors. 
Shifting to studies on the speech patterns of minority language speakers in bilingual societies, the fourth article, "Sociolinguistic awareness in Galician bilinguals: Evidence from an accent identification task" by Gisela Tomé Lourido (Leeds University) and Bronwen G. Evans (University College London), assesses the extent to which a new variety of Galician spoken by Galician new speakers (so-called neofalantes) is distinctive enough to be identifiable by listeners in an accent identification task. The results revealed, however, that while Galician-dominant bilinguals and to a lesser extent Spanish-dominant bilinguals could be clearly identified, listeners from Galicia were unable to classify neofalantes correctly. Instead, the latter were categorized as both Galician- and Spanish-dominant, suggesting the variety constitutes a hybrid accent. The authors argue that listeners may have a gradient representation of variation in which the accents of Galician-dominant and Spanishdominant speakers function as anchors, with neofalantes' accent situated in between. The results also revealed differences in identification accuracy across listener groups, with neofalantes exhibiting particular sensitivity to the accents of Galician-dominant speakers. This may be due to neofalantes' heightened awareness of the sociolinguistic landscape in Galicia and their motivation to learn Galician.

The fifth article, "Adult new speakers of Welsh: Accent, pronunciation and language experience in South Wales" by Meinir Williams and Sarah Cooper (Bangor University), investigates the experiences of adult new speakers of Welsh with little to no exposure to the language in childhood, with learning the pronunciation patterns of Welsh. Using an online questionnaire, the study examined how respondents perceived their Welsh accent, which speech sounds they found most challenging and how traditional speakers responded to them. The results revealed that the respondents generally did not perceive their accent as native-like, and that perceptions of their accent depended on their level of competence in Welsh, with beginning and intermediate learners considering their accent as less native, being less proud of their accent and wanting to change their accent more than advanced or fluent learners. They indicated that vowel length and consonants that are not shared with English were most challenging. Finally, the study revealed a range of reactions by traditional speakers, including switching to English, decreasing speaking rate or correcting the respondents' pronunciations. These findings have important implications for new speakers' participation in the community and for language learning.

The sixth article, "Social influences on phonological transfer: / $\mathrm{r} /$ variation in the repertoire of Welsh-English bilinguals" by Jonathan Morris (Cardiff University), is also situated within the context of bilingualism in Wales. Specifically, it examines the effects of speaker gender, home language and speech context on the Welsh and English /r/ productions of bilingual speakers from two communities in North Wales, Caernarfon and Mold, that differ in their use of Welsh as a community language. Data collected from young Welsh-English bilinguals via sociolinguistic interviews and word lists revealed cross-linguistic and areal differences. Thus, Welsh /r/ variants, such as the trill and tap, occurred substantially more in the Welsh than the English productions of Caernarfon speakers, and were entirely absent in the English data from Mold. Moreover, in contrast to previous dialectological studies, alveolar approximants were found to be widely used in Welsh in both communities, suggesting cross-linguistic transfer from English. This pattern was more pronounced in new speakers than traditional ones, showing an effect of home language. Effects were also found for task and speaker gender. Together, the study reveals a complex pattern in which the realization of $/ \mathrm{r} /$ in the two languages is mediated by a multifaceted interplay of different social variables, reflecting sociolinguistic differences across the two communities and differences in peer group structure.

Shifting to studies examining L1 and L2 speech and its interrelation in bilingual adults, the next article, "Psycho-social constraints on naturalistic adult second language acquisition" by Azza Al-Kendi (Sultan Qaboos University) and Ghada Khattab (Newcastle University), investigates the speech perception and production patterns in L2 Arabic of Foreign Domestic Helpers (FDH) in Omani homes with a range of native languages. As such, it is one of few studies on bilinguals from low educational backgrounds who learn an 
L2 predominantly, if not solely, to interact with their employers. The results from an AX discrimination task revealed low sensitivity to Arabic consonantal contrasts that do not exist in the participants' L1 while the results of a production task showed low accuracy on Arabic consonants. Moreover, in an accent rating task, L1 Omani Arabic speakers identified a marked foreign accent in the FDH's speech, in particular in those that were not literate in Arabic. Interestingly, unlike previous studies on L2 speech, length of residence was not found to be a significant predictor of performance in any of the tasks. The authors argue that this is due to the specific social context of FDH and the unequal power relations between them and their employers.

The eighth article, "The effect of dual language activation on L2-induced changes in L1 speech within a code-switched paradigm" by Ulrich Reubold (Karl-Franzens-Universität Graz), Sanne Ditewig (Karl-Franzens-Universität Graz), Robert Mayr (Cardiff Metropolitan University) and Ineke Mennen (Karl-Franzens-Universität Graz), examined the L1 English speech of adult migrants to Austria in a code-switched and monolingual condition alongside that of monolingual English speakers in England. The code-switched materials involved German words with segments known to trigger cross-linguistic interactions being inserted into an otherwise English frame; the monolingual materials, in turn, contained the equivalent segments in wholly English sentences. The sentences were produced in an online reading task and the critical items subsequently analyzed acoustically. The results revealed no differences between the monolingual and bilingual speakers in the monolingual condition. However, on some sounds significant L2-induced shifts in L1 speech production were observed in the code-switched condition. These occurred both before and after the switch. On the other hand, with one exception, i.e., amount of L2 use for $[w]$, none of the predictor variables examined were associated with L2-induced shifts across conditions. These results have significant implications for the role of dual activation in the pronunciation patterns of late bilinguals.

In "Foreign-language phonetic development leads to first-language phonetic drift: Plosive consonants in native Portuguese speakers learning English as a foreign language in Brazil" by Denise M. Osborne (State University of New York at Albany) and Miquel Simonet (University of Arizona), word-initial plosive productions in Portuguese were compared across L1 Brazilian Portuguese speakers learning English as an L2 in Brazil and monolingual native speakers of Brazilian Portuguese. The L2 learners' English plosive productions were also investigated and compared with their L1 categories. The results of an acoustic analysis of the participants' voice onset time (VOT) patterns revealed first that the learners produced Portuguese voiced plosives with a longer voicing lead than the monolinguals. They also showed that the learners' English plosives were similar to their Portuguese ones, showing cross-linguistic interactions but also some phonetic development. Together, the study adds to the growing literature on bidirectional interactions in bilinguals' speech and shows that these can even occur outside L2 immersion contexts.

The final article, "Longitudinal developments in bilingual second language acquisition and first language attrition of speech: The case of Arnold Schwarzenegger" by Lisa Kornder and Ineke Mennen (Karl-Franzens-Universität Graz), investigates the speech productions of the late consecutive bilingual Arnold Schwarzenegger in his L1 (Austrian German) and L2 (English) over a period of 40 years. As such, it is the first longitudinal case study that examines bilingual speech development over several decades in both languages. Specifically, on the basis of speech samples from broadcast interviews, it measured the VOT durations in his plosive productions and the formant frequencies in his vowel productions across different time periods. The results revealed a cross-linguistic merger of $/ \mathrm{p} \mathrm{t} \mathrm{k} /$ in his late productions. His vowel realizations in the two languages also became increasingly more similar. These findings shed new light on bilinguals' reorganization of L1 and L2 sound systems over time and their dynamic interrelations.

Taken together, the ten articles in this Special Issue significantly advance our understanding of the role that different social and psychological factors play in the speech productions of bilinguals across a wide range of settings, and have important theoretical 
implications. They also highlight important future directions in this vibrant field of enquiry, which, we hope, will inspire others in their pursuit of new knowledge.

Acknowledgments: First and foremost, we would like to thank the authors of the articles in the Special Issue for their fine contributions. It has been a great pleasure to work with each of them. We are also most grateful to the international set of experts who agreed to act as reviewers for the articles in this volume and helped to improve the quality of the contributions. In alphabetical order, these are John Archibald (University of Victoria), Elise Bell (University of California, Los Angeles), Gwen Brekelmans (University College London), Chiara Celata (Università degli Studi di Urbino Carlo Bo), Ulrike Gut (Westfälische Wilhelms-Universität Münster), Mike Hammond (University of Arizona), Michael Hornsby (Adam Mickiewicz University, Poznań), Lisa Kornder (Karl-Franzens-Universität Graz), Rosangela Lai (Università di Pisa), Chia-Cheng Lee (Portland State University), Philip P. Limerick (New Mexico State University), Diarmait Mac Giolla Chríost (Cardiff University), Daniela Mereu (Free University of Bozen-Bolzano), Alene Moyer (University of Maryland), Naomi Nagy (University of Toronto), Ramsés Ortín (University of Texas, Rio Grande Valley), Michael Putnam (Pennsylvania State University), Yasaman Rafat (Western University), Anabela Rato (University of Toronto), Xosé-Luis Regueira Fernández (Universidade de Santiago de Compostella), Monika Schmid (University of Essex), Geoffrey Schwartz (Adam Mickiewicz University, Poznań), and Antje Stoehr (Basque Center on Cognition, Brain \& Language). Last but not least, we would like to thank the team at Languages, in particular Yuki Yu and Milana Arambašić, for their editorial support.

Conflicts of Interest: The authors declare no conflict of interest.

\section{References}

Best, Catherine T., and Michael Tyler. 2007. Nonnative and second-language speech perception: Commonalities and complementarities. In Language Experience in Second Language Speech Learning, in Honor of James Emil Flege. Edited by Ocke-Schwen Bohn and Murray J. Munro. Amsterdam: John Benjamins, pp. 13-24.

Escudero, Paola. 2005. Linguistic Perception and Second Language Acquisition. Utrecht: Utrecht University.

Flege, James E., and Ocke-Schwen Bohn. 2021. The revised Speech Learning Model. In Second Language Speech Learning, Theoretical and Empirical Progress. Edited by Ratree Wayland. Cambridge: Cambridge University Press, pp. 3-83.

Mayr, Robert, Jonathan Morris, Ineke Mennen, and Daniel Williams. 2017. Disentangling the effects of long-term language contact and individual bilingualism: The case of monophthongs in Welsh and English. International Journal of Bilingualism 21: $245-67$. [CrossRef]

Nance, Claire. 2020. Bilingual language exposure and the peer group: Acquiring phonetics and phonology in Gaelic medium education. International Journal of Bilingualism 24: 360-75. [CrossRef]

Piske, Thorsten, Ian R. A. MacKay, and James E. Flege. 2001. Factors affecting degree of foreign accent in an L2: A review. Journal of Phonetics 29: 191-215. [CrossRef]

Sharma, Devyani, and Lavanya Sankaran. 2011. Cognitive and social forces in dialect shift: Gradual change in London Asian speech. Language Variation and Change 23: 399-428. [CrossRef] 